\title{
Eight-Hit Evolutionary Pattern in ATM Gene of a Breast Carcinoma Patient: A Personalized Approach
}

\author{
Parvin Mehdipour ${ }^{1, *}$ and Asaad Azarnezhad ${ }^{1,2}$ \\ ${ }^{1}$ Department of Medical Genetics, School of Medicine, Tehran University of Medical Sciences, Tehran, Iran \\ ${ }^{2}$ Department of Molecular Medicine and Medical Genetics, Kurdistan University of Medical Sciences, \\ Sanandaj, Iran
}

\begin{abstract}
Background: Different genetic variants in the ATM gene have been reported to be associated with breast carcinoma (BC). Purpose of the study was to consider the multi-insights experiments and explorations through the molecular, cellular and structural aspects of ATM.

Methods and Results: D1853N polymorphism was traced in a proband with BC. DNA extracted from blood and tumor tissue was PCR-amplified and cloned to allelic localization of characterized variants. Protein expression and In silico analysis based on three different profiles involved in cell cycle were also performed to confirm mutational events. Findings revealed the molecular based sequential events as an eight-hit evolutionary pattern (8- Hit E.P) including $\mathrm{D} 1853 \mathrm{~N}$ as the first predisposing hit (inherited), pre-differentiation stage hits including IVS $36-8 \mathrm{~T}>\mathrm{C}$ as the $2^{\text {nd }}$ hit, V1833M as the $3^{\text {rd }}$ hit, L1888L as the $4^{\text {th }}$, and somatic variants including IVS $36-46 \mathrm{C}>\mathrm{T}, \mathrm{L} 1842 \mathrm{~L}, \mathrm{H} 1864 \mathrm{H}$, and S1872R were considered as the $5-8^{\text {th }}$ hits. Low protein expression of ATM in the majority of cells was observed, but the expression of cyclin E, CDC25A, P53, and Ki-67 was more diverse.

Conclusions: Observations were reflective of the sequential molecular and cellular events through the entire patient's life from the pre-differentiation embryonic stage and all through the post-birth periods. Mentioned hits seem to be effective on expression and function of ATM which confirmed by the expression and in silico analysis.
\end{abstract}

Keywords: Ataxia Telangiectasia Mutated gene (ATM), Breast carcinoma, Hit-Evolutionary pattern, Hypothesis, Protein expression, In silico, Personalized management, innovative therapy.

\section{INTRODUCTION}

The malignant breast cells are formed through the multi-developmental episodes including the programmed proliferation accompanied by a different cascade of events to guarantee the occurrence of breast carcinoma (BC). BC is reported as the most common malignancy within women in almost all of the geographical regions [1]. A variety of factors including physiological, environmental, and family history $(\mathrm{FH})$ are involved in the development of BC [2]. Several genetic factors have been recognized to be linked with BC susceptibility. However, BRCA1/BRCA2, p53, PTEN, STK11/LKB1, CDH1, ATM, CHECK2, BRIP1, and PALB2 genes are highlighted as the most important genetic risk factors in $B C$ patients $[1,3]$.

Ataxia telangiectasia-mutated (ATM) gene is located at chromosome11q22-q23 providing a $13 \mathrm{~kb}$ transcript instructions for making a $350 \mathrm{kDa}$ serine/threonine protein kinase. ATM is recruited to the sites of double strand breaks (DSBs) by Mre11-Rad50Nbs1 (MRN) complex and helps cells recognize damaged or broken DNA through activation of cell

*Address correspondence to this author at the Department of Medical Genetics, School of Medicine, Tehran University of Medical Sciences, Tehran, Iran; Tel/Fax: +0098-2188830100; E-mail: mehdipor@tums.ac.ir cycle checkpoint elements including P53, NBS1, CHEK2, and BRCA1. Hence, it is known as the first sensing component of DNA damage [4]. The loss of function of ATM directly leads to Ataxia telangiectasia (AT), furthermore, carriers of ATM mutations have a higher risk of developing $\mathrm{BC}$. Accordingly, we have reported that the missense substitution of aspartic acid with asparagine in codon $1853(\mathrm{D} 1853 \mathrm{~N})$ is noticeably more frequent in $\mathrm{BC}$ patients than the control groups [5]. Besides, the D1853N could be detected either in healthy individuals or carcinoma patients.

As a micro-evolutionary process, initial hit in the key genes such as oncogenes, tumor suppressors, and repair genes could increase the chance of different mutation acquiring in cells by conferring a growth advantage or by inducing genomic instability [6].The real Hit introduced by knudson refers to a sequential/molecular alteration in a specific gene and in its specific exon and intron known as two-hit hypothesis in development of retinoblastoma [7-9]. The multi-hits could occur at different stages including perdifferentiation, through the whole embryonic period, post-birth and through the individual's life. However, a predisposing element, such as D1853N polymorphism in our case is hypothesized to be essential for initiation and promotion of HIT- process [9]. 
Our aim was based on; 1) The critical role of $\mathrm{D} 1853 \mathrm{~N}$ polymorphism; 2) tracing $\mathrm{D} 1853 \mathrm{~N}$ and possibly the occurrence of evolutionary hits in a $\mathrm{BC}$ patient; 3 ) our previous publications on the importance of $\mathrm{D} 1853 \mathrm{~N}$ ( $\mathrm{rs} 1801516$ ) in the primary BC patients [5], and 4) formulating our three-hit hypothesis [8]. Cooperation, harmonic behavior, restriction, supporting, aggressiveness are examples of the multi-paradigm insights which are rather confusing in carcinoma. As the final aim, combating against carcinoma as a challenging issue requires multi-directional insights. Therefore, design of this project was based on the multi-insight experiments and exploration through the molecular/cellular/structural aspects of ATM gene as a frame of triangle support for our findings.

\section{MATERIALS AND METHODS}

\section{Patient's Information}

A pedigree-based investigation was performed on a female proband, aged 42 years old, affected with infiltrating ductal carcinoma (IDC) of the right breast who was referred to the unit of Cancer Genetics, Tehran University of Medical Sciences. The tumor was characterized with grade III, size of 4.5. Centimeters, and 28/45 auxiliary lymph nodes were revealed to be metastatic. Furthermore, the fibrocystic changes of non-tumoral breast tissues were reported. Core tissue biopsies were provided in size of $2 \times 3 \times 4 \mathrm{~cm}$ for downstream analysis. The current study has been approved by the Research Ethic Committee of Tehran University of Medical Science (Tehran, Iran) and a signed informed consent form (according to the Declaration of Helsinki) was taken from probands. The comprehensive pedigree was drawn by the cancer genetic counselor through multi-counselling and face to face interview (S. Figure 1).

\section{Pedigree Analysis}

There was no $\mathrm{FH}$ of $\mathrm{BC}$ in her paternal or maternal sides, but her first son was affected with right $\mathrm{BC}$ at age of 41-years old. The $\mathrm{FH}$ of other malignancy in the relatives of our proband included second cousin with brain tumor at age of 20 years who deceased few months later, her mother was affected with lymphoma at age of 63 years who deceased at the age of 74 , and the proband's far paternal relative with benign uterus neoplasm at the age of 60 and deceased 10 years later (S. Figure 1).

\section{Design of Study}

The design of this investigation was based on; 1) An early age of onset of the proband, 2) the pedigree- based insight to record the confirmed information and documentation of the informative key facts, 3) multisampling approach including the peripheral blood and other tissues, and 4) considering the molecular and cell-based strategies. This study has been designed and performed at Department of Medical Genetics, School of Medicine, Tehran University of Medical Sciences, Tehran, Iran.

\section{Preparation of DNA, Polymerase Chain Reaction (PCR), and Sequencing}

Total DNA was extracted from proband's tumor tissue and her peripheral blood by the phenol/chlorophorm method. The PCR reaction was run in Veriti® Thermal Cycler (Applied Biosystems, USA) according to the previous report [8]. Primer sequences used to amplify the target fragment of ATM were Exon-37F (5'- TTTAATATGTCAACGGGGCAT 3') and Exon-37R (5' CCATCTTAAATCCATCTTTCTC $\left.-3^{\prime}\right)$, generating products were of $342 \mathrm{bp}$. PCR products was directly sequenced (ABI 3130XL Genetic analyzer).

\section{Molecular Cloning}

The PCR products were purified using PCR Cleanup Kit (Roche, Germany). Purified PCR products were cloned into PTZ57R (T) vector using InsTAclone Kit (Fermentas, Lithuania) according to manufacturer's instruction. Selection of recombinants was performed as previously reported [8-10]. Plasmid extractions were performed from recombinant colonies, extracted plasmids were electrophoresed on $1 \%$ agarose gel, and those showing $342 \mathrm{bp}$ larger size than control (vector without insert) were sequenced.

\section{Two-Dimension (2D) and Three-Dimension Structure Prediction of Mutant ATM}

(3D)

Impact of molecular alterations found in proband on secondary (2D) and tertiary (3D) structure of ATM protein was analyzed using bioinformatics tools including PSIPRED, PHD, and Jpred 4 (for secondary structure prediction), I-TASSER, VADAR, Galaxy refine, TM-align and SuperPose (for $3 \mathrm{D}$ prediction).A part of the ATM protein sequence containing exon 37 (NP_000042.3.) was selected and the prediction was carried out based on this region. The effect of nucleotide changes on the splicing at the acceptor site was evaluated by NetGen2 software from CBS prediction services and Human Splice Finder (HSF) system. 


\section{Immunofluorescence (IF) Assay}

The process of cell extraction, and conjugation was performed according to the previous publications [13, 14]. Three relevant profiles were considered for the IF assay: Profile A): Includes: 1. the mouse monoclonal anti-Cyclin $\mathrm{E}$ antibody, isotype $\lg \mathrm{g} 2 \mathrm{~b}$ (Invitrogen, USA); goat anti-mouse; 2 . Monoclonal mouse anti-ATM antibody, isotype lgG1 (CEDARLANE, Hornby, Ontario, Canada) with goat anti-mouse (GE/Amersham Biosciences); and 3. monoclonal anti-CDC25A (Sigma, USA), with second layer antibody goat anti-mouse (GE/Amersham Biosciences), Profile B: Includes: 1. Monoclonal mouse anti-human PCNA, conjugated isotype IgG2a/FITC (DakoCytomation, Denmark); 2. The monoclonal anti-P53 antibody, isotype $\lg \mathrm{G} 2 \mathrm{~b}$ (DakoCytomation, Denemark) and 3. Monoclonal mouse anti-ATM antibody, isotype $\operatorname{lgG} 1$. Profile $\mathbf{C}$ : Includes: 1. Monoclonal mouse anti-human Ki-67, conjugated with FITC, 2. Mouse monoclonal anti-Cyclin D1 antibody, with Rpe, and 3. The monoclonal anti-P53 antibody, isotype lgG2b, with /Pe-cy5.The corresponding mouse immunoglobulin (Dako, Denmark) was used as negative control for the same population of each triple profile. Three blood samples were used as controls which included a sample of the proband and two samples from the healthy subjects in the pedigree who were negative for ATM-D1853N alteration.

\section{RESULTS}

\section{Molecular Data}

In addition to D1853N polymorphism, the sequencing results of blood and tumor tissues revealed two intronic mutations including IVS 36-46 C>T and IVS 36-8 T>C located within the donor splice site of intron 36 and five coding alterations of $\mathrm{V} 1833 \mathrm{M}$ and L1888L, L1842L, H1864H, and S1872R (Figure 1a, b). All the alterations were observed hetrerozygously in the proband (Figure 1b). D1853N was traced through the pedigree which found to be hetrerozygously inherited from proband's mother, while the other heterozygote alterations were somatic variations observed in tumor and blood samples of the proband. Furthermore, D1853N polymorphism was also positive in the proband's son who is affected with BC at age of 41 .

\section{Cloning Results}

D1853N occurred in two different alleles of the proband. Furthermore, both D1853N, IVS 36-8 T>C, $\mathrm{V} 1833 \mathrm{M}$, and L1888L presented in both blood- and tumor- tissue (Figure 1a, b). The other hit- events including IVS 36-46 T>C, L1842L, $\mathrm{H} 1864 \mathrm{H}$, and S1872R were, heterzygously, observed at tumor level, and not in blood sample (Figure 1b). H1864H, S1872R, and L1888L were observed in different allele from allele

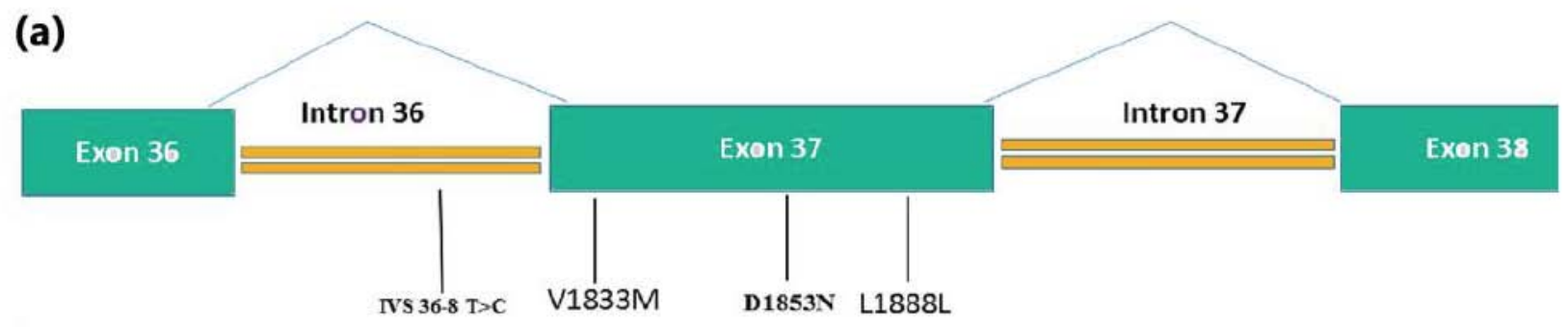

(b)

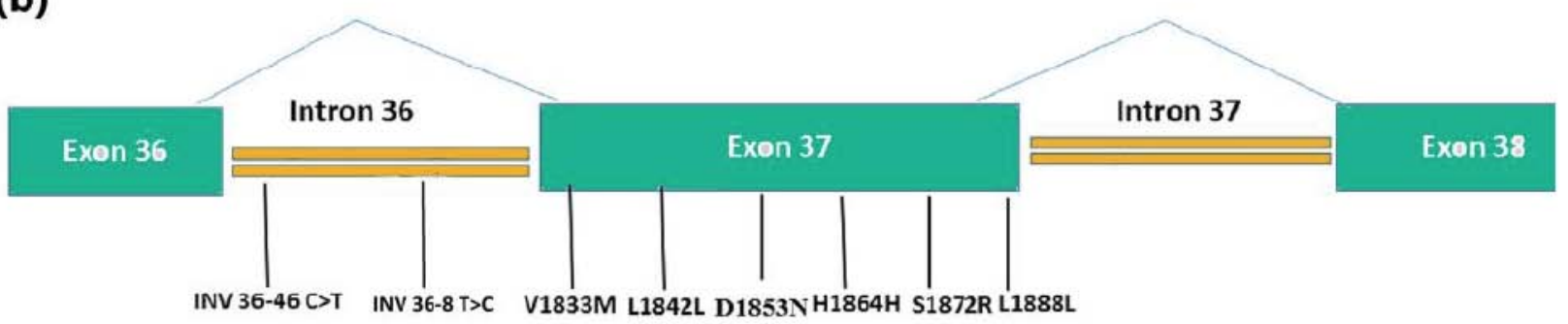

Figure 1: The alterations in Exon 37 and surrounding region of ATM gene.

1a. Sequencing results of blood and tumor tissues present two intronic mutations including IVS 36-46 C>T and IVS 36-8 T> within the splice site of intron 36 and the coding alterations includes V1833M and L1888L, L1842L, H1864H, and S1872R.

1b. Alterations are heterozygous in the proband.

D1853N, IVS 36-8 T>C, V1833M, and L1888L presented in blood- and tumor- tissue. IVS 36-46 T>C, L1842L, H1864H, and S1872R were, traced in tumor $\mathrm{H} 1864 \mathrm{H}, \mathrm{S} 1872 \mathrm{R}$, and L1888L were observed in different allele. 

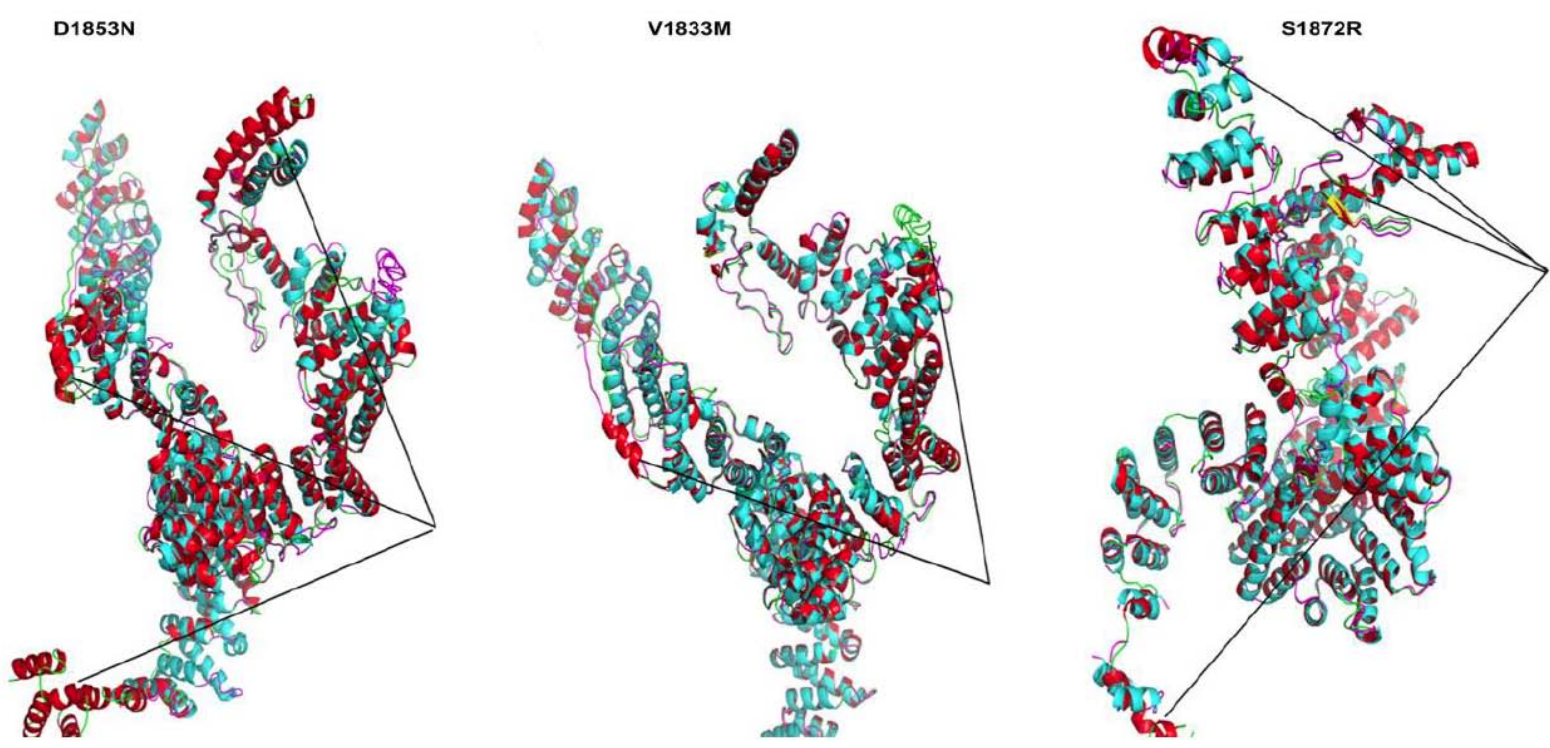

Figure 2:The 3D structure prediction of target region of ATM protein.

The in silico analysis showed that each alteration of $\mathrm{V} 1833 \mathrm{M}, \mathrm{D} 1853 \mathrm{~N}$, and S1872R changes the distribution of helixes and loops in the modelled region of ATM. Arrows are indicative of the possible differences introduced by V1833M, D1853N, and S1872R alterations on the 3D-conformation of modelled region.

containing IVS 36-46 T>C, IVS 36-8 T>C, V1833M, L1842L, and D1853N hit events.

\section{In Silico Protein Structure Prediction}

The 2D prediction tools showed that coding alterations consisted of D1853N, V1833M, and $\mathrm{S} 1872 \mathrm{R}$. The 2D prediction tools showed that they cause 2D structure alteration in ATM protein through disturbing the percentage and size of shits, helixes and coils. Consistent with 2D, 3D structure alignment of wild-type and mutant-type revealed that the mentioned alterations obviously convey conformational differences on the ATM protein structure (Figure 2). In silico analysis by NetGen2 showed that IVS 36-46 T>C could decrease the probability of using the main splice site from $33 \%$ to $26 \%$. Also, IVS $36-46$ T>C was found to increases the probability of an alternative splice site. HSF matrices predicted that the substitutions of $\mathrm{T}$ with C-allele and C with T-allele result in the loss of $3^{\prime}$ splice donor site with decreasing the consensus value.

Modification of two-hit hypothesis of tumor suppressor gene and inactivation of E-cadherin [11], and the key role of ATM in development of breast cancer [12] are indicative of the evolving role in molecular cell biology.

\section{Immunofluorescence (IF)}

The results of protein expression (PE) assay were provided by three different profiles. Profile A reflects the PE of Cyclin E, ATM and CDC25A (Figure 3); Profile B includes PCNA, P53 and ATM (Figure 4); and profile C comprises of Ki-67, Cyclin D1 and P53 (S. Figure 4). The follow-up expression assay of cyclin $E$, ATM and CDC25A in the breast tumor of our proband was, initially, performed the same day after surgery (Figure 3), and followed by the buccal assay after 21 years (S Figure 5).

As profile $A$ reflects, the PE of ATM is low in the majority of cells, but of cyclin $E$ and CDC25A is more diverse (Figure $3 \mathbf{a}-\mathbf{h}$ ). In Profile-B, expression of ATM is low in the majority of cells, and the degree of intensity is diverse in PCNA and P53 (Figure 5a-g). In profile $\mathrm{C}, \mathrm{Ki}-67$, cyclin $\mathrm{D} 1$, and $\mathrm{P} 53$ had a diverse expression ( $S$. Figure $\mathbf{4 a - d}$ ). The PE is found to be high in cyclin $\mathrm{E}$ and CDC25A (Figure 3a-h), and the high expression of cyclin E, ATM, and CDC25A in buccal cells of proband after 21 years of the initial surgery as a primary IDC is remarkable (Figure 4).

\section{DISCUSSION}

Carcinoma occurs as a result of the sequential events at genomic and somatic levels including molecular and functional alterations. It is believed that at least 3-7 genetic alterations are required for carcinoma development [9]. There are a few reports where in those the hit evolutionary molecular based pattern in BC has been evaluated, but not in ATM [1012]. Somatic mutation, loss of heterozygosity ( $\mathrm{LOH})$, and methylation have been reported as the hit 
a

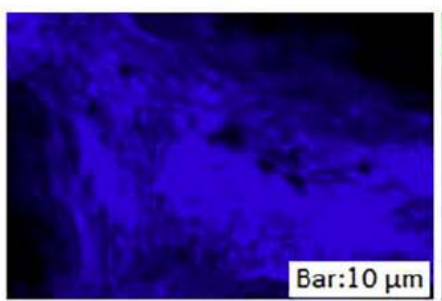

e

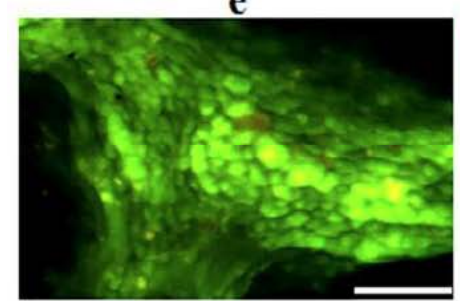

b

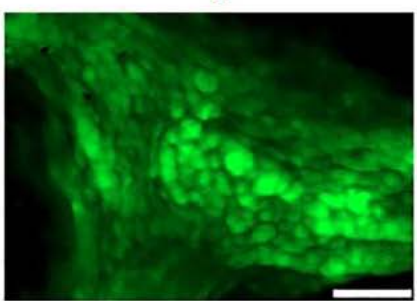

f

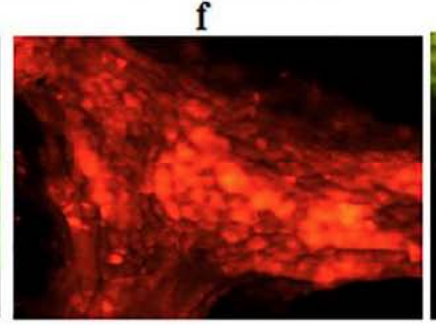

c

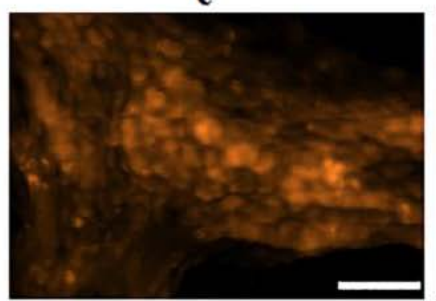

$\boldsymbol{\sigma}$

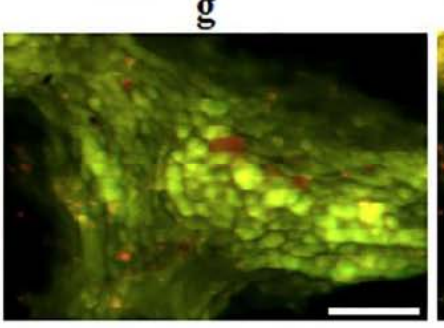

i

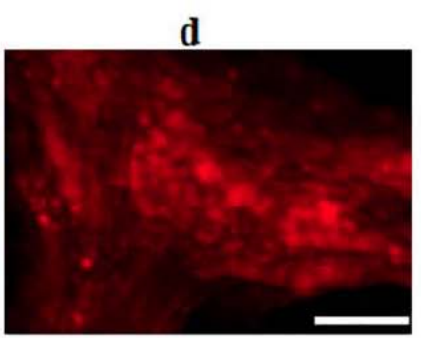

h

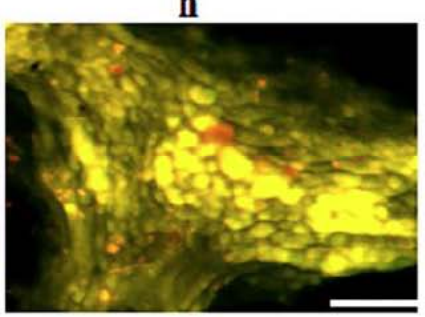

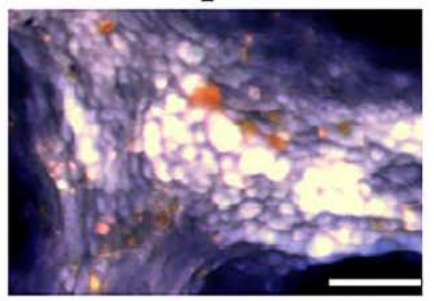

Figure 3: "Protein expression of Cyclin E, ATM and CDC25A in tumor of a patient with breast carcinoma.

a) Breast tumor cells with dapi; b) The same cells conjugated with FITC, reflecting the expression of Cyclin E; c) The same cells conjugated with Rpe, reflecting the expression of ATM; d) The same cells presenting the expression of CDC25A and conjugated with Pe-cy5; e) The co-expression of Cyclin E/ATM; $\mathbf{f}$ ) the co-expression of cyclin ATM/ CDC25A; $\mathbf{g}$ ) The co-expression of Cyclin E/CDC25A; $\mathbf{h}$ ) the co-expression of Cyclin E/ATM/CDC25A; i) The co-expression of dapi/Cyclin E/ATM/CDC25A. Diverse expression and remarkable co-expression is observable.

a

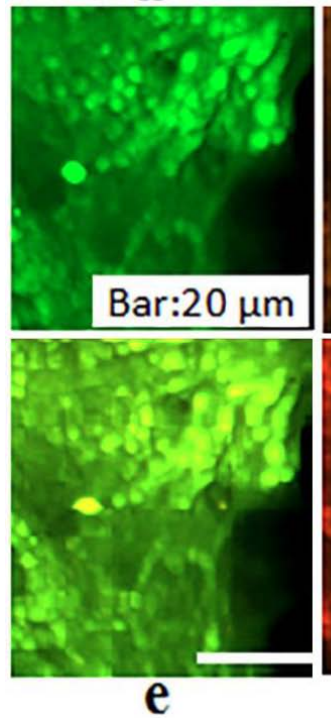

b

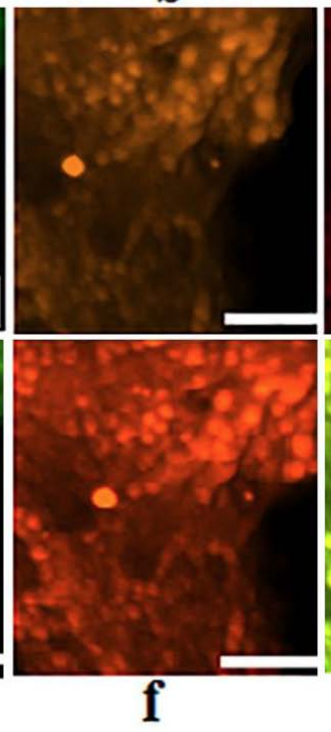

C

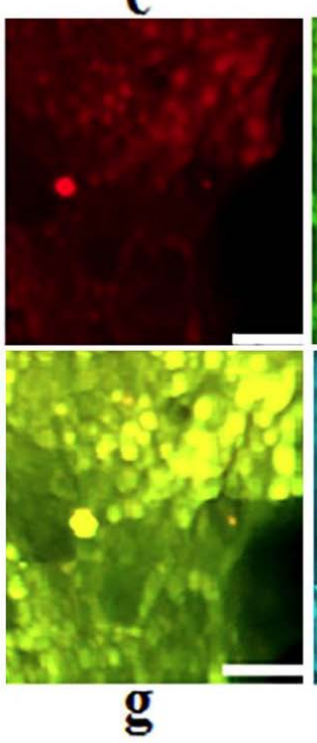

d

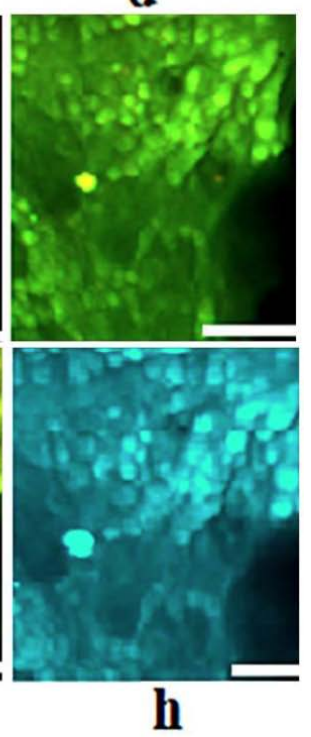

Figure 4: Protein expression of PCNA, P53 and ATM in the tumor of a patient with Breast carcinoma.

a) Breast tumor cells conjugated with FITC, reflecting the expression of PCNA; b) The same cells conjugated with Rpe, reflecting the expression of P53; c) The same cells conjugated with Pe-cy5, reflecting the expression of ATM; d), The same cells presenting the co-expression of PCNA /P53; e) The co-expression of PCNA /ATM; $\mathbf{f}$ ) The co-expression of P53/ATM; g) The coexpression of PCNA /P53/ATM; $\mathbf{h}$ ) The merged of dapi/P53/ATM

Diverse expression (a-c) and co-expression (d-g) is observable. 


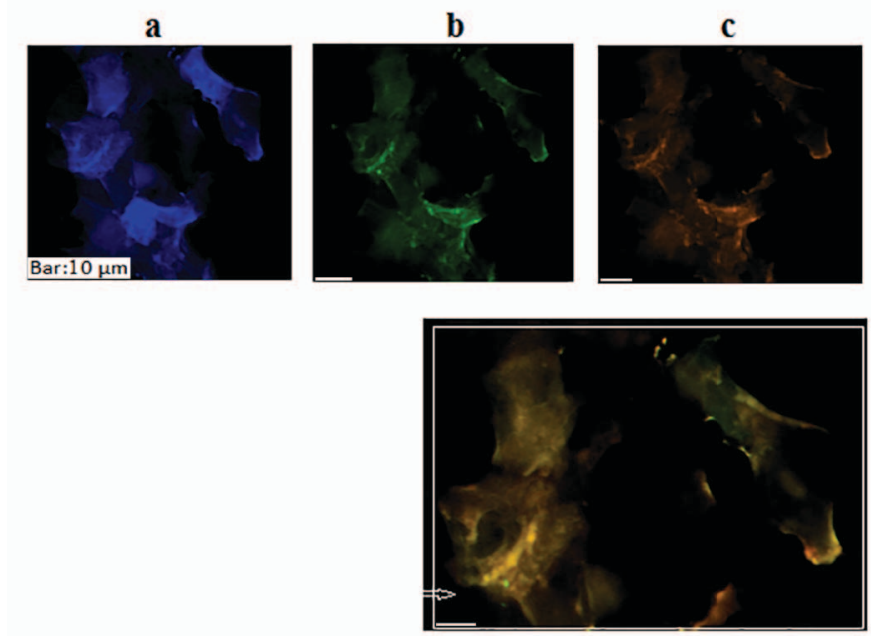

g
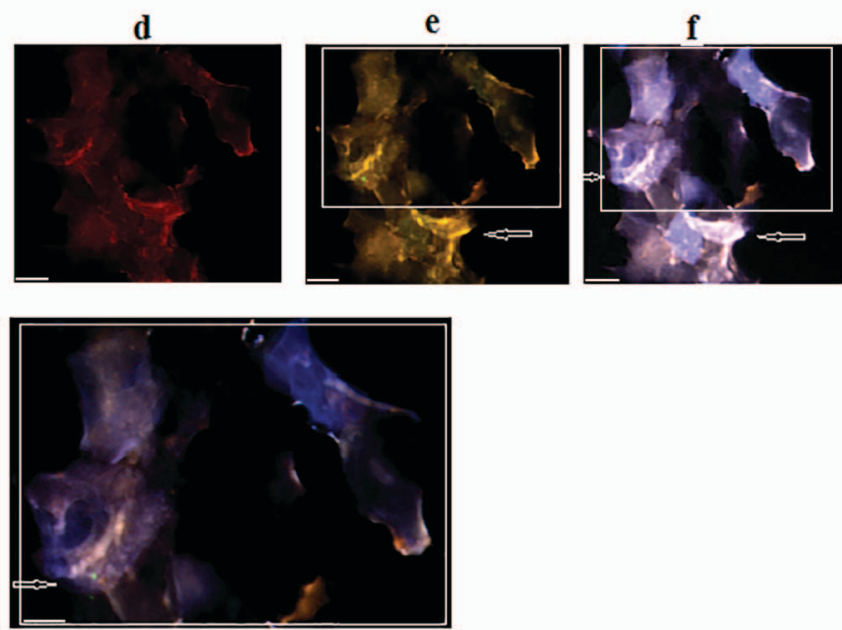

h

Figure 5: Status of protein expression of Cyclin E, ATM and CDC25A in buccal in a patient affected with breast carcinoma at the same day before surgery

Arrows show the high expression and co-expression of Cyclin E and CDC25A in limited buccal cells 21 years after surgery.

a) Buccal cells with dapi; b) The same cells conjugated with FITC, reflecting the expression of Cyclin E; c) The same cells conjugated with Rpe, reflecting the expression of ATM; d), The same cells presenting expression of CDC25A and conjugated with Pe-cy5; e) The co-expression of Cyclin E/ATM/CDC25A in cropped image from; f) The co-expression of dapi/Cyclin E/ATM/CDC25A; g) The cropped section from image e, reflect the co-expression of Cyclin E/ATM/CDC25A; $\mathbf{h}$ ) The cropped section from image $\mathbf{f}$, reflects the co-expression of dapi/ Cyclin E/ATM/CDC25A.

contributed to the loss of $A T M$ function, however, a real hit is reflective of alteration (s) occurring in a gene and the same related exon [13]. The involvement of ATM gene and specifically, the key role of D1853N polymorphism, has been previously reported in different types of carcinomas especially $\mathrm{BC}$ and this polymorphism could be screened in a variety of carcinomas [5, 13]. A patent has been also registered by Mehdipour (Application number: PCT/IB2014/ 065072). The D1853N polymorphism has been highlighted as a predisposing variant [8]. Furthermore, a five-hit hypothesis could be traced in a patient affected with an early onset breast carcinoma [14]. In this patient, five heterozygous variants including IVS 36-91 AA>TT, IVS 36-8 T>C, D1853N, IVS $37+47$ $A>G$, IVS $37+60$ Del T were found in the exon 37 and its splicing site of ATM.

In the current study, we traced D1853N alteration in a proband with $\mathrm{BC}$ and evaluated the molecular evolution of hits occurred in exon 37 and its flanking region in ATM. The results unmasked the occurrence of the evolutionary alterations through the process of tumorigenesis. At a glance, our findings are reflective of the eight hits including IVS 36-46 C>T, IVS 36-8 T>C, V1832M, L1842L, D1853N (occurred in an allele), $\mathrm{H} 1864 \mathrm{H}, \mathrm{S} 1872 \mathrm{R}$ (at somatic level) and L1888L (occurred at genomic and somatic level in other allele) in target region of $A T M$ of our proband.
In addition to the inherited D1853N polymorphism, the alterations including IVS $36-8 \mathrm{~T}>\mathrm{C}, \mathrm{V} 1833 \mathrm{M}$, and L1888L were found to be occurred before the differentiation of blood system at early developmental stage, at embryonic period of the proband, i.e., between the beginning of $2^{\text {nd }}$ and the end of $3^{\text {rd }}$ weeks after fertilization of oocyte (S. Figure 6). Interestingly, the tumor-specific presence of IVS 36-8 T>C, V1833M, and $\mathrm{L} 1888 \mathrm{~L}$ alterations is indicative of the key role of these evolutionary changes through the BC progression either at genomics or tumor level in our patient. This phenomenon has also been previously reported in the three-hit hypothesis in astrocytoma [8]. Surprisingly, further evolution has occurred at tumor level which was found as IVS 36-46 C>T, L1842, $\mathrm{H} 1864 \mathrm{H}$, and S1872R, characterized as the $5^{\text {th }}, 6^{\text {th }}, 7^{\text {th }}$ and $8^{\text {th }}$ hit events, respectively. The key elements of evolution, is shown in supplementary Figure $\mathbf{2}$ and Supplementary Figure 3. Apart from the D1853N polymorphism and those alterations which were only observed in breast tumor, the further hit events observed both in blood and tumor tissues of proband could be considered as the early events at a very early stage of differentiation. Therefore, we may conclude that this stage of life is very critical which is, partly, related to the lifestyle of the proband's mother and other factors including the predisposing capability of the proband. 
In overview, either environmental factors during the proband's embryonic life, or genetic susceptibility of a triangle partner including parents or embryo could be highlighted. In this case, the influential advice is essential to be provided to the couples. These are the matter of an early systematic and applicable strategy which seems to be the key elements for carcinoma management. In the previous report conducted by Mehdipour and her colleagues, the heterozygous D1853N polymorphism was proposed as an essential molecular alteration for successful evolutionary events in the astrocytoma. Furthermore, two additional intronic hit events at the splicing site of ATM gene at Exon 37 were aimed to progress the tumorigenic event by completing the evolutionary process [5]. The probability of pathogenic effects of reported hit events was analyzed in silico based on some bioinformatics tools such as SIFT, PolyPhen, MUpro, and Project HOPE. According to the COSMIC catalogue and bioinformatics tools, D1853N is considered as a predisposing coding variant that is located at a crucial region and decreases the stability of protein structure. The mutant amino acid has a different charge that can cause loss of interactions with other molecules or residues. However, this genetic alteration is predicted to be benign based on the dbSNP (https://www.ncbi.nlm.nih.gov/snp/ rs1801516), but with a predisposing capability. Our clinical and molecular findings also confirmed the pathogenic effects, therefore, $\mathrm{D} 1853 \mathrm{~N}$ is considered as an initiator hit to support tumorigenesis of deficient ATM in proband which is confirming the previous reports [15-17]. There were two other missense alterations including V1833M and S1872R. According to in silico analysis, these alterations are predicted to be "POSSIBLY DAMAGING" due to the differences in size, charge, and hydrophobicity between wild-type and mutant amino acids. Supporting the mentioned data, comparison of 2D (data are not presented) and 3D structure model of the selected region containing D1853N, V1833M and S1872R showed an obvious change between the wild-type allele and the mutant one, that consequently might introduce function deficiency and pathological effects (Figure 1b).

Since the number of each tRNAs related to each amino acid codon is not equal in the tRNA pool of the cell, therefore, a synonymous change could also have an adverse impact on the speed and level of translation and consequently the level of protein production. This phenomenon is reflected "as codon usage bias" [18, 19]. Accordingly, substitution of CTT with CTC (L1842L), substitution of CAT with CAC $(\mathrm{H} 1864 \mathrm{H})$, and substitution of CTT with CTC (L1888L) may have an inverse impact on ATM protein production and finally exert the pathological effect. Interestingly, the ATM gene harbor the predisposition capability to be evolved with more aggressive behavior than an oncogene.

In addition to the invariant $5^{\prime}-\mathrm{GT}$ and $3^{\prime}$-AG splicing sites, the extensive consensus sequence spanning $5^{\circ}$ and $3^{\prime}$ ' splice sites is also a determining factor of an accurate splicing $[20,21]$. Consequently, variations affecting splicing are expected to compromise a noticeable proportion of genetic disease etiology [22]. IVS 36-8 T>C (rs3092829) and IVS 36-46 C>T were the splicing region variants. Although the most straightforward and reliable method to detect splicing defects is the analysis of RNA, in silico analysis by NetGen2 and HSF matrices disclosed the adverse effect of these alterations on splice efficacy and consequently, these intronic hit events can have pathological impacts as well.

Regarding the in silico analysis, predicted score should be interpreted with caution and further in vitro studies are required which may address the possible impact of coding and splicing variants on function and expression of ATM protein [23]. The mitotic cell cycle is progressed through a reproducible sequence of events that are regulated by different kinds of proteins. When Eukaryotic cells are exposed to DNA damage, a signaling pathway will be directed by ATM through which the activation or inactivation of the involved genes including $P 53$ in either $G 1$ or $G 2$ would occur. Besides, degradation of CDC25A protein phosphatase, as an intra-S-phase checkpoint consequence, is considered as a key event during the cell cycle [24].

Focusing on the functional insight, it was previously reported that the PE of CDC25A and cyclin E were significantly correlated with Ki-67 expression level [25]. Furthermore, no clear data on the co-expression and impact of these three genes on the clinical outcome of $B C$ are available. However, it is expected that the status of PE in CDC25A, Cyclin D1, and cyclin E would remain high, but PE in P53 will be constantly low in carcinoma cells. In addition, due to the $\mathrm{FH}$ of the brain tumor in proband's second cousin, the issue of $\mathrm{Li}$ Fraumeni's syndrome was considered, however, no mutation was detected in P53.

In our patient, regarding the profile-A, the low PE of ATM in the majority of cells confirms the diverse molecular alterations as eight-hit. However, cyclin E and $C D C 25 \mathrm{~A}$ revealed to have more diverse pattern of PE (Figure 3a-i). 
Furthermore, the co-expression between proteins of ATM/CDC25A was remarkable for few cells, but more harmonic cooperation is detectable between cyclin $\mathrm{E}$ and CDC25A (Figure 3d) which is facilitating more aggressive behavior of the $\mathrm{BC}$ tumor. These functional data are due to the diverse molecular alteration and the priority of essential and required genes for the survival of $B C$ cells. This explanation is also indicative of a severe heterogeneity at the cellular level.

In Profile- $B$, degree of intensity in majority of cells is revealed to be high for PCNA and moderate in P53 which is indicative of the high proliferation and the relative influential role of P53 as the cell guardian respectively. But, the PE of $A T M$ is low in the majority of cells with a harmonic co-expression with P53 (Figure 3a-d). Besides, there is a prominent single cell with harmonic triangle cooperation due to the high expression of ATM and P53 which may have an influential positive impact on the tumor status by restricting and/or balancing the progression of $B C$ tumor by PCNA (Figure $\mathbf{4 b} \mathbf{b}-\mathbf{d}$ ). In spite of the occurrence of an evolutionary eight- hit events and based on the current 21 years' survival period of the proband, the presence of a minor clone, with a normal function of ATM and higher expression of P53, had a remarkable positive impact on the health status and a satisfactory quality of her life.

In Profile-C, Ki-67, cyclin D1, and P53 have a diverse expression, however Ki-67 and cyclin D1 reflect a harmonic cooperation (S. Figure 4). Although the expression of P53 is low in this profile, some cell population with high PE may indicate the resistance of P53 to rescue the target cells from more invasive behavior (S. Figure 4d).

Furthermore, the initial assay of PE in the buccal cells of the proband indicated the high expression of cyclin $E$ and CDC25A in few cells with a negative impact on the course of disease in our BC patient (Figure 5a-h). The follow-up study on her buccal cells reflects the diverse and low expression of ATM, cyclin $E$, and CDC25A after 21 years of the initial surgery of the proband but with noticeable co-expression (S. Figure 5a-f).

In conclusion, our findings reflect the sequential molecular and cellular events through the entire patient's life from the pre-differentiation embryonic stage and all through the post-birth periods. In spite of such events, our patient is also living in a stable psychological status. Finally, these data are reflective of a personalized diagnosis that would lead to manage the early preventive strategies, either for the patient or for the relatives of proband. Moreover, as such hit events may occur in other carcinoma patients, similar studies may be applied as a screening test for an appropriate personalized carcinoma management and may lead to an innovative therapy.

\section{FUNDING}

This research has received any grant neither from any funding agency in the public, nor from the commercial / profit sectors.

\section{CONFLICT OF INTEREST}

The authors declare that they have no conflict of interest.

\section{ETHICS CERTIFICATE}

Ethics approval code: IR.TUMS.Medicine.Rec.1395. 1893.

Project number: 95-04-30-32208

Principal investigator: Professor Parvin Mehdipour

Approval date: 08/03/2017

\section{CONSENT TO PARTICIPATE}

A signed informed consent form (according to the Declaration of Helsinki) was taken from proband.

\section{AUTHORS' CONTRIBUTION}

Parvin Mehdipour contributed to the design of study; analyses of data; configuring the molecular sequential cascade, and manuscript edition. Asaad Azarnezhad was involved in laboratory works, data analysis, bioinformatics analysis, and manuscript writing.

\section{COMPETING INTERESTS}

The authors disclose any financial or non-financial interests that are directly or indirectly related to the present work submitted for possible publication.

\section{SUPPLEMENTARY FIGURE}

The supplementary Figures can be downloaded from the journal website along with the article.

\section{REFERENCE}

[1] Karami F, Mehdipour P. A comprehensive focus on global spectrum of BRCA1 and BRCA2 mutations in breast cancer. BioMed Research international 2013. https://doi.org/10.1155/2013/928562 
[2] Yang XR, Chang-Claude J, Goode EL, et al. Associations of breast cancer risk factors with tumor subtypes: a pooled analysis from the Breast Cancer Association Consortium studies. Journal of the National Cancer Institute 2011; 25063.

https://doi.org/10.1016/j.yonc.2011.10.003

[3] Azarnezhad A, Mehdipour P. Cancer Genetics at a Glance: The Comprehensive Insights. Cancer Genetics and Psychotherapy. Springer 2017; pp. 79-389. https://doi.org/10.1007/978-3-319-64550-6 5

[4] Sandoval N, Platzer M, Rosenthal A, et al. Characterization of ATM gene mutations in 66 ataxia telangiectasia families. Human Molecular Genetics 1999; 69-79. https://doi.org/10.1093/hmg/8.1.69

[5] Mehdipour P, Mahdavi M, Mohammadi-Asl J, Atri M. Importance of ATM gene as a susceptible trait: predisposition role of D1853N polymorphism in breast cancer. Medical Oncology 2011; 733-7.

https://doi.org/10.1007/s12032-010-9525-0

[6] Alberts B, Johnson A, Lewis J, Raff M, Roberts K, Walter P. Cancer as a microevolutionary process 2002.

[7] Knudson AG. Mutation and cancer: statistical study of retinoblastoma. Proceedings of the National Academy of Sciences 1971; 820-3.

https://doi.org/10.1073/pnas.68.4.820

[8] Mehdipour P, Habibi L, Mohammadi-AsI J, Kamalian N, Azin MM. Three-hit hypothesis in astrocytoma: tracing the polymorphism D1853N in ATM gene through a pedigree of the proband affected with primary brain tumor. Journal of Cancer Research and Clinical Oncology 2008; 1173-80. https://doi.org/10.1007/s00432-008-0404-4

[9] Frank SA. Dynamics of cancer: incidence, inheritance, and evolution. Princeton University Press 2007.

https://doi.org/10.1515/9780691186863

[10] Yang Q, Nakamura M, Nakamura $Y$, et al. Two-hit inactivation of FHIT by loss of heterozygosity and hypermethylation in breast cancer. Clinical Cancer Research 2002; 2890-3.

[11] Cheng C-W, Wu P-E, Yu J-C, et al. Mechanisms of inactivation of E-cadherin in breast carcinoma: modification of the two-hit hypothesis of tumor suppressor gene. Oncogene 2001; 3814-23.

https://doi.org/10.1038/sj.onc. 1204505

[12] Prokopcova J, Kleibl Z, Banwell CM, Pohlreich P. The role of ATM in breast cancer development. Breast Cancer Research and Treatment 2007; 121-8. https://doi.org/10.1007/s10549-006-9406-6

[13] Mehdipour P. The final words: the cyclic bridging programme for the cancer clinic. Bridging cell biology and genetics to the cancer clinics Transworld network, India 2011. https://doi.org/10.2741/e828

[14] Mehdipour P, Azarnezhad A. Five-hit hypothesis in ATM gene: An individualized model in a breast cancer patient. Frontiers in Bioscience (Elite edition) 2018; 375-83.
[15] Izatt L, Greenman J, Hodgson S, et al. Identification of germline missense mutations and rare allelic variants in the ATM gene in early-onset breast cancer. Genes, Chromosomes and Cancer 1999; 286-94. https://doi.org/10.1002/(SICl)10982264(199912)26:4<286::AID-GCC2>3.0.CO;2-X

[16] Thorstenson YR, Roxas A, Kroiss R, et al. Contributions of ATM mutations to familial breast and ovarian cancer. Cancer Research 2003; 3325-33.

[17] Rahman N. Realizing the promise of cancer predisposition genes. Nature 2014; 302-8. https://doi.org/10.1038/nature12981

[18] Van Weringh A, Ragonnet-Cronin M, Pranckeviciene E, Pavon-Eternod M, Kleiman L, Xia X. HIV-1 modulates the tRNA pool to improve translation efficiency. Molecular Biology and Evolution 2011; 1827-34. https://doi.org/10.1093/molbev/msr005

[19] Kimchi-Sarfaty C, Oh JM, Kim I-W, et al. A" silent" polymorphism in the MDR1 gene changes substrate specificity. Science 2007 ; 525-8. https://doi.org/10.1126/science.1135308

[20] Krawczak M, Reiss J, Cooper DN. The mutational spectrum of single base-pair substitutions in mRNA splice junctions of human genes: causes and consequences. Human Genetics 1992; 41-54. https://doi.org/10.1007/BF00210743

[21] Linde L, Boelz S, Neu-Yilik G, Kulozik AE, Kerem B. The efficiency of nonsense-mediated mRNA decay is an inherent character and varies among different cells. European Journal of Human Genetics 2007; 1156-62. https://doi.org/10.1038/sj.ejhg.5201889

[22] Jian X, Boerwinkle E, Liu X. In silico tools for splicing defect prediction: a survey from the viewpoint of end users. Genetics in Medicine 2013; 497-503. https://doi.org/10.1038/gim.2013.176

[23] Richards S, Aziz N, Bale S, et al. Standards and guidelines for the interpretation of sequence variants: a joint consensus recommendation of the American College of Medical Genetics and Genomics and the Association for Molecular Pathology. Genetics in Medicine 2015; 405-23. https://doi.org/10.1038/gim.2015.30

[24] Branzei D, Foiani M. Regulation of DNA repair throughout the cell cycle. Nature Reviews Molecular Cell Biology 2008; 297308. https://doi.org/10.1038/nrm2351

[25] Mehdipour P, Pirouzpanah S, Sarafnejad A, Atri M, Shahrestani ST, Haidari M. Prognostic implication of CDC25A and cyclin E expression on primary breast cancer patients. Cell Biology International 2009; 1050-6. https://doi.org/10.1016/j.cellbi.2009.06.016

\section{https://doi.org/10.30683/1929-2279.2021.10.04}

() 2021 Mehdipour and Azarnezhad; Licensee Neoplasia Research.

This is an open access article licensed under the terms of the Creative Commons Attribution Non-Commercial License (http://creativecommons.org/licenses/by-nc/3.0/) which permits unrestricted, non-commercial use, distribution and reproduction in any medium, provided the work is properly cited. 